\title{
AN INVARIANCE PROBLEM FOR CONTROL SYSTEMS WITH DETERMINISTIC UNCERTAINTY
}

\author{
LECH GÓRNIEWICZ \\ Institute of Mathematics, University of N. Copernicus \\ ul. Chopina 12/18, 87100 Toruń, Poland \\ E-mail: gorn@mat.uni.torun.pl \\ PAOLO NISTRI \\ Dipartimento di Sistemi e Informatica, Università degli Studi di Firenze \\ Via di S. Marta 3, 50139 Firenze, Italy \\ E-mail: pnistri@ingfi1.ing.unifi.it
}

\begin{abstract}
This paper deals with a class of nonlinear control systems in $\mathbf{R}^{n}$ in presence of deterministic uncertainty. The uncertainty is modelled by a multivalued map $F$ with nonempty, closed, convex values. Given a nonempty closed set $K \subset \mathbf{R}^{n}$ from a suitable class, which includes the convex sets, we solve the problem of finding a state feedback $\bar{u}(t, x)$ in such a way that $K$ is invariant under any system dynamics $f$. As a system dynamics we consider any continuous selection of the uncertain controlled dynamics $F$.
\end{abstract}

0. Introduction. In this paper the invariance property of a given nonempty, closed set $K \subset \mathbf{R}^{n}$ under a nonlinear controlled dynamics is investigated. The dynamics of the control system is affected by a deterministic uncertainty which is modelled by a multivalued map $F$. Here we consider only the continuous selections $f$ of $F$ as possible system dynamics. Specifically, we consider a differential inclusion of the form

$$
\dot{x} \in F(t, x, u), t \in[0, T], u \in U \subset \mathbf{R}^{m}, x \in \mathbf{R}^{n} .
$$

Given a nonempty, closed set $K \subset \mathbf{R}^{n}$, the problem that we want to study is that of determining a state feedback control $\bar{u}(t, x)$ such that $K$ is invariant under any corresponding system dynamics $f(t, x, \bar{u}(t, x)) \in F(t, x, \bar{u}(t, x))$. That is, for any initial state $x_{0} \in K$, if $x(t)$ is a solution of the Cauchy problem

$$
\left\{\begin{array}{l}
\dot{x}=f(t, x, \bar{u}(t, x)) \quad \text { for a.a. } t \in[0, T], \\
x(0)=x_{0},
\end{array}\right.
$$

1991 Mathematics Subject Classification: 93C10, 93C15, 93B52.

The paper is in final form and no version of it will be published elsewhere. 
then $x(t) \in K$ for each $t \in[0, T]$. In this paper the approach to solve this problem is based on the viability theory and the relative invariance property of a set. Therefore, we make use both of the contingent Bouligand cone $T_{K}(x)$ and of the external contingent cone $\widehat{T}_{K}(x)$ to the set $K$ at a point $x$. Then, we define the regulation map as $R(t, x)=$ $\left\{u \in U: F(t, x, u) \subset T_{K}\left(\pi_{K}(x)\right)\right\}$ for $(t, x) \in[0, T] \times \mathbf{R}^{n}$, where $\pi_{K}(x)$ is the continuous projection of $x$ onto $K$. The control $\bar{u}(t, x)$ will be a selection of this map.

The problem in doing this is that, in general, the feedback control $\bar{u}(t, x)$ turns out to be only measurable and so not enough regular to allow to consider classical solutions of (2), i.e. absolutely continuous functions $x(t), t \in[0, T]$, satisfying (2) for almost all (a.a.) $t \in[0, T]$. For this, we have to introduce regularizations of $f$. We consider here the Krasovskiiı's regularization and the Filippov's regularization. Unfortunately, when we consider the regularizations of $f$ it is not more guaranteed that they are still contained in the Bouligand tangent cone. To overcome this difficulty we make suitable assumptions on the set $K$ and on the uncertain dynamics $F$ in order to obtain a more regular feedback control $\bar{u}(t, x)$ as selection of $R(t, x)$. Specifically, we will obtain a directionally continuous map $\bar{u}(t, x)$ with respect to a certain cone $\Gamma^{M}$ in $\mathbf{R} \times \mathbf{R}^{n}$. For this, we use a selection theorem due to Bressan ([10], Theorem 1$)$. The $\Gamma^{M}$-continuity property of $\bar{u}(t, x)$ is the main tool for obtaining the desired invariance property. In fact, by this notion we can show that the solution set of (2) and that of the Krasovskil's regularization coincide. Then we solve our problem by proving that any solution of (2) is viable.

Obviously, if the assumptions on the set $K$ and the uncertain dynamics $F$ guarantee that the corresponding regulation map $R(t, x)$ fulfills the assumption of the celebrated Michael's selection theorem then there exists a Carathéodory feedback control $\bar{u}(t, x) \in R(t, x)$ and we can proceed in the usual way (compare [1], [2], [3], [13], [16], $[21])$. Therefore, our results will generalize those obtained in the previous references in the singlevalued case.

Several control problems require a viability or an invariance property of some subset $K$ of the state space. For instance, control problems with state constraints, observability and tracking problems. In this paper our approach is based on the viability theory. Other approaches, based on Liapunov functions have been also used. In [19] and [20] a non smooth Liapunov function together with the calculus for non differentiable functions were employed in order to solve the problem of the asymptotic linearization of a nonlinear uncertain dynamical system. This problem was considered also in [7] and [8]. In this case the approach relies to the theory of Variable Structure Systems.

When the control appears linearly and the uncertainty is modelled as a perturbation of the nominal plant, rather than by a differential inclusion, several authors (see e.g. [5], [6], [12] and [15]) designed, by techniques based on smooth Liapunov functions, a continuous robust feedback controller guaranteeing some stability requirements on the system. In [4], a system under uncertainty governed by a differential inclusion is considered and criteria for different types of local observability are given.

Finally, for an overview on the relevant and interesting work performed by the Russian school in the field of uncertain dynamical system we refer to [17] and [18] and the extensive references therein. The relevant tool for modelling the uncertain dynamics is represented 
by the theory of trajectory tubes for differential inclusions. Several problems concerning systems with constraints on the state have been solved by means of this theory.

1. Statement of the problem and some preliminaries. We consider a nonlinear control system with deterministic uncertain dynamics modelled by a differential inclusion of the form:

$$
\dot{x} \in F(t, x, u), t \in[0, T], u \in U \subset \mathbf{R}^{m}, x \in \mathbf{R}^{n}
$$

where $U$ is a connected compact set.

Throughout the paper a system dynamics $f$ will be a continuous function $f: D:=$ $[0, T] \times \mathbf{R}^{n} \times U \rightarrow \mathbf{R}^{n}$ such that $f(t, x, u) \in F(t, x, u)$ for any $(t, x, u) \in D$.

We assume the following conditions on the dynamics $F: D \multimap \mathbf{R}^{n}$.

$\left(\mathrm{H}_{1}\right) F(t, x, u)$ is a nonempty closed convex set for any $(t, x, u) \in D, F$ is $(t, x, u)$ continuous in the Hausdorff metric.

Observe that, by $\left(\mathrm{H}_{1}\right)$ and the Michael's selection theorem, the set of the system dynamics $f$ is non empty.

Formulation of the problem. Given a nonempty closed set $K \subset \mathbf{R}^{n}$. To find a state feedback control $\bar{u}(t, x)$ defined in $[0, T] \times \mathbf{R}^{n}$ such that for every dynamics $f(t, x, \bar{u}(t, x)) \in F(t, x, \bar{u}(t, x))$ and every $x_{0} \in K$ any solution $x(t), t \in[0, T]$, of the Cauchy problem

$$
\left\{\begin{array}{l}
\dot{x}=f(t, x, \bar{u}(t, x)), \\
x(0)=x_{0},
\end{array}\right.
$$

satisfies $x(t) \in K$ for each $t \in[0, T]$, i.e. it is viable. In other words, $\bar{u}(t, x)$ makes $K$ invariant under every dynamics $f(t, x, \bar{u}(t, x))$.

Since, in general, the control law $(t, x) \rightarrow \bar{u}(t, x)$ will not be continuous in the variable $x$, but only measurable in the pair $(t, x)$ we cannot expect that $(2)$ possesses classical solutions, i.e. absolutely continuous function $x(t)$ satisfying $(2)$ for a.a. $t \in[0, T]$. This is the reason why we will consider two regularizations of the function $g(t, x):=f(t, x, \bar{u}(t, x))$, called respectively the Krasovskiı's regularization and the Filippov's regularization of $g$, (see [2]). They are defined as follows:

$$
\begin{aligned}
& K(g)(t, x)=\bigcap_{\delta>0} \overline{\operatorname{co}} g(B((t, x), \delta)), \\
& F(g)(t, x)=\bigcap_{\delta>0} \bigcap_{\mu(N)=0} \overline{\operatorname{co}} g(B((t, x), \delta)-N),
\end{aligned}
$$

where $\mu$ denotes the Lebesgue measure in $\mathbf{R}^{n+1}, \overline{\text { co }}$ the closed convex hull and $B((t, x), \delta)$ the open ball centered at the point $(t, x)$ with radius $\delta$.

Any absolutely continuous solution of the multivalued Cauchy problems

$$
\begin{array}{ll}
\dot{x} \in K(g)(t, x), & x(0)=x_{0}, \\
\dot{x} \in F(g)(t, x), & x(0)=x_{0},
\end{array}
$$

on the time interval $[0, T]$ is called a Krasovskiı's (Filippov's) solution to (2).

Obviously any Filippov's solution is a Krasovskiı's solution. Thus, if we solve the proposed problem for (3) we have solved the problem also for system (4). 
In what follows we will provide conditions on the dynamics $F$ and on the set $K$ which together $\left(\mathrm{H}_{1}\right)$ will ensure that any solution of $(3)$ is viable, whenever $x_{0} \in K$. For this, we give the following:

Definition 1 ([1], [2], [3]). Let $K$ be a nonempty closed subset of $\mathbf{R}^{n}$. The set $T_{K}(x) \subseteq \mathbf{R}^{n}, x \in K$, defined by

$$
T_{K}(x)=\left\{y \in \mathbf{R}^{n} \mid \liminf _{\tau \rightarrow 0^{+}} \frac{1}{\tau} \operatorname{dist}(x+\tau y, K)=0\right\}
$$

where $\operatorname{dist}(u, K)=\inf \{|u-x| \mid x \in K\}$, is called the Bouligand contingent cone to $K$ at $x \in K$.

Our problem requires that $K \subset \mathbf{R}^{n}$ is invariant under any dynamics $f(t, x, \bar{u}(t, x))$ $\in F(t, x, \bar{u}(t, x)),(t, x) \in[0, T] \times \mathbf{R}^{n}$. The invariance property involves the behaviour of $F$ outside of $K$. Therefore we need to extend the notion of contingent cone to $K$ to a set $A \supset K$. For this purpose, following [1] we introduce the concept of external contingent cone to $K$.

Definition 2. Let $K \subset A$ be a non empty closed set of $\mathbf{R}^{n}$. Let $x \in A$, the set $\widehat{T}_{K}(x) \subseteq \mathbf{R}^{n}$ defined by

$$
\widehat{T}_{K}(x)=\left\{y \in \mathbf{R}^{n} \mid \liminf _{\tau \rightarrow 0^{+}} \frac{1}{\tau}[\operatorname{dist}(x+\tau y, K)-\operatorname{dist}(x, K)] \leq 0\right\}
$$

is called the external contingent cone to $K$ at the point $x \in A$.

Obviously, $\widehat{T}_{K}(x)=T_{K}(x)$ for any $x \in K$. Let $x \in A$, denote by $\Pi_{K}(x)$ the set of projections of $x$ onto $K$, i.e. the set of points $z \in K$ such that $|x-z|=\operatorname{dist}(x, K)$. We have the following result:

Proposition 1 ([1], Lemma 5.1.2). Let $K$ be a non empty closed subset of $\mathbf{R}^{n}$. Then

$$
T_{K}\left(\Pi_{K}(x)\right) \subseteq \widehat{T}_{K}(x) .
$$

We introduce now a particular class of nonempty closed sets $K$ by means of the following definition.

Definition 3 ([1], [3]). A nonempty closed subset $K \subseteq \mathbf{R}^{n}$ is said to be sleek if the multivalued map $T_{K}: K \multimap \mathbf{R}^{n}$ is lower semicontinuous.

It is well known that any nonempty closed convex set $K$ is sleek ([3], Theorem 4.2.2). Furthermore if $K$ is sleek then the multivalued map $T_{K}: K \multimap \mathbf{R}^{n}$ is convex-valued ([3], Theorem 4.1.8). A larger class of sleek sets is represented by the so-called proximate etracts (see [9], [23]).

Definition 4. A nonempty closed subset $K$ of $\mathbf{R}^{n}$ is said to be a proximate retract if there exists an open neighbourhood $I$ of $K$ in $\mathbf{R}^{n}$ and a continuous map $r: I \rightarrow K$ (called metric retraction) such that the following two conditions are verified.

$$
\begin{aligned}
r(x) & =x & & \text { for each } x \in K \\
|r(y)-y| & =\operatorname{dist}(y, K) & & \text { for each } y \in I .
\end{aligned}
$$

It is known that any $C^{2}$-manifold of $\mathbf{R}^{n}$ is a proximate retract (see [23]).

We can now formulate the following assumptions. 
$\left(\mathrm{H}_{2}\right)$ There exists $\gamma>0$ such that for any $(t, x) \in[0, T] \times \mathbf{R}^{n}$ there exists $u \in U$ for which

$$
F(t, x, u)+\gamma B_{1} \subset T_{K}\left(\Pi_{K}(x)\right),
$$

where $B_{1}$ is the unitary open ball centered at the origin.

$\left(\mathrm{H}_{3}\right)$ For any $(t, x) \in[0, T] \times \mathbf{R}^{n} ; u, v \in U$ and $\theta \in[0,1]$ there exists $\widetilde{u} \in U$ such that

$$
\theta F(t, x, u)+(1-\theta) F(t, x, v)=F(t, x, \widetilde{u}) .
$$

Furthermore, there exist constants $a, b>0$ such that

$$
a|u-v| \leq d(F(t, x, u), F(t, x, v)) \leq b|u-v|
$$

for any $(t, x) \in[0, T] \times \mathbf{R}^{n}$ and any pair $u, v \in U$.

Here $d(A, B)$ denotes the Hausdorff distance of the two sets $A, B$. We recall that $d$ is a metric in the family of all nonempty, bounded and closed subsets of a Banach space.We give the following.

Definition 5. The multivalued map $R:[0, T] \times \mathbf{R}^{n} \multimap U$ defined as follows:

$$
R(t, x)=\left\{u \in U: F(t, x, u) \subset T_{K}\left(\Pi_{K}(x)\right)\right\}
$$

is called the regulation map.

In the sequel we will denote $T_{K}\left(\Pi_{K}(x)\right)$ by $T(x)$.

Finally, we assume the following condition.

$\left(\mathrm{H}_{4}\right) \Pi_{K}: \mathbf{R}^{n} \rightarrow K$ is a singlevalued, continuous function, which will be denoted by $\pi_{K}$.

We are now in a position to prove the following.

Theorem 1. Let $K \subseteq \mathbf{R}^{n}$ be a sleek subset of $\mathbf{R}^{n}$. Assume $\left(\mathrm{H}_{1}\right)-\left(\mathrm{H}_{4}\right)$. Then the regulation map $R$ has nonempty closed values and it is lower semicontinuous.

Proof. The proof follows the lines of that of ([2], Theorem 3, p. 49), where a singlevalued autonomous dynamics, affine in the control, was considered.

In virtue of $\left(\mathrm{H}_{2}\right)$ the regulation map $R(t, x)$ has nonempty values for any $(t, x) \in$ $[0, T] \times \mathbf{R}^{n}$. Furthermore, $R(t, x)$ is closed by $\left(\mathrm{H}_{1}\right)$. We have now to prove that for fixed $(t, x) \in[0, T] \times \mathbf{R}^{n}, u \in R(t, x)$ and $\varepsilon>0$ there exists a neighbourhood $N(t, x)$ of $(t, x)$ such that for any $\left(t^{\prime}, x^{\prime}\right) \in N(t, x)$ there exists $\widetilde{u} \in R\left(t^{\prime}, x^{\prime}\right) \cap\left(u+\varepsilon B_{1}\right)$.

Let $\delta>\operatorname{diam} U$ such that $\delta>\left(2 \gamma+\varepsilon^{\prime} a\right) / 2 a$, with $\varepsilon^{\prime}=2 a \varepsilon /(a+b)$. Let $\alpha=$ $\gamma \varepsilon^{\prime} /\left(2 \delta-\varepsilon^{\prime}\right)>0$. Since $F$ is continuous in the Hausdorff metric, $U$ is compact and $T$ is lower semicontinuous there exists a neighbourhood $N(t, x)$ of $(t, x)$ and $\eta<\varepsilon^{\prime}$ such that for any $\left(t^{\prime}, x^{\prime}\right) \in N(t, x)$ we have:

1. for any $u_{1}, u_{2}$ with $\left|u_{1}-u_{2}\right|<\eta$ we have $d\left(F\left(t^{\prime}, x^{\prime}, u_{1}\right), F\left(t, x, u_{2}\right)\right) \leq \alpha / 2$;

2. $F(t, x, u) \subset T\left(x^{\prime}\right)+(\alpha / 2) B_{1}$.

Therefore, for any $\left(t^{\prime}, x^{\prime}\right) \in N(t, x)$ there exists $v^{\prime} \in U$ with $\left|u-v^{\prime}\right|<\eta$ such that

$$
F\left(t^{\prime}, x^{\prime}, v^{\prime}\right) \subset T\left(x^{\prime}\right)+\alpha B_{1} .
$$


Let $0<\theta<\gamma /(\alpha+\gamma)<1$, since $\theta \alpha=(1-\theta) \gamma$ by multiplying (5) by $\theta$ we obtain

$$
\theta F\left(t^{\prime}, x^{\prime}, v^{\prime}\right) \subset \theta T\left(x^{\prime}\right)+(1-\theta) \gamma B_{1} .
$$

By $\left(\mathrm{H}_{2}\right)$ we have that for any $\left(t^{\prime}, x^{\prime}\right) \in N(t, x)$ there exists $u^{\prime} \in U$ such that

$$
(1-\theta) F\left(t^{\prime}, x^{\prime}, u^{\prime}\right)+(1-\theta) \gamma B_{1} \subset(1-\theta) T\left(x^{\prime}\right) .
$$

Summing (6) and (7) we get

$$
\begin{aligned}
\theta F\left(t^{\prime}, x^{\prime}, v^{\prime}\right)+(1-\theta) F^{\prime}\left(t^{\prime}, x^{\prime}, u^{\prime}\right) & +(1-\theta) \gamma B_{1} \\
& \subset \theta T\left(x^{\prime}\right)+(1-\theta) T\left(x^{\prime}\right)+(1-\theta) \gamma B_{1},
\end{aligned}
$$

by the convexity of $T\left(x^{\prime}\right)$ we have

$$
\theta F\left(t^{\prime}, x^{\prime}, v^{\prime}\right)+(1-\theta) F\left(t^{\prime}, x^{\prime}, u^{\prime}\right)+(1-\theta) \gamma B_{1} \subset T\left(x^{\prime}\right)+(1-\theta) \gamma B_{1} .
$$

Thus

$$
\theta F\left(t^{\prime}, x^{\prime}, v^{\prime}\right)+(1-\theta) F\left(t^{\prime}, x^{\prime}, u^{\prime}\right) \subset T\left(x^{\prime}\right) .
$$

By $\left(\mathrm{H}_{3}\right)$ there exists $\widetilde{u} \in U$ such that

$$
\theta F\left(t^{\prime}, x^{\prime}, v^{\prime}\right)+(1-\theta) F\left(t^{\prime}, x^{\prime}, u^{\prime}\right)=F\left(t^{\prime}, x^{\prime}, \widetilde{u}\right),
$$

that is $\widetilde{u} \in R\left(t^{\prime}, x^{\prime}\right)$. Now to finish the proof we must show that $|u-\widetilde{u}|<\varepsilon$. For this, we consider

$$
d\left(F(t, x, u), F\left(t^{\prime}, x^{\prime}, \widetilde{u}\right)\right)=d\left(F(t, x, u), \theta F\left(t^{\prime}, x^{\prime}, v^{\prime}\right)+(1-\theta) F\left(t^{\prime}, x^{\prime}, u^{\prime}\right)\right) .
$$

From the properties of the Hausdorff metric (see e.g. [16]) we obtain

$$
\begin{aligned}
d\left(F(t, x, u), F\left(t^{\prime}, x^{\prime}, \widetilde{u}\right)\right) \leq \theta d(F(t, x, u), & \left.F\left(t^{\prime}, x^{\prime}, v^{\prime}\right)\right) \\
& +(1-\theta) d\left(F(t, x, u), F\left(t^{\prime}, x^{\prime}, u^{\prime}\right)\right) .
\end{aligned}
$$

On the other hand

$$
d\left(F(t, x, u), F\left(t^{\prime}, x^{\prime}, u^{\prime}\right)\right) \leq d\left(F(t, x, u), F\left(t, x, u^{\prime}\right)\right)+d\left(F\left(t, x, u^{\prime}\right), F\left(t^{\prime}, x^{\prime}, u^{\prime}\right)\right) .
$$

By using again $\left(\mathrm{H}_{3}\right)$ we obtain

$$
d\left(F(t, x, u), F\left(t^{\prime}, x^{\prime}, \widetilde{u}\right)\right) \leq \frac{\alpha}{2}+(1-\theta) b\left|u-u^{\prime}\right| \leq \frac{\alpha}{2}+b \frac{\varepsilon^{\prime}}{2},
$$

since $(1-\theta)\left|u-u^{\prime}\right| \leq \varepsilon^{\prime} / 2$. But

$$
\begin{aligned}
d\left(F(t, x, u), F\left(t^{\prime}, x^{\prime}, \widetilde{u}\right)\right) & \geq d(F(t, x, u), F(t, x, \widetilde{u}))-d\left(F(t, x, \widetilde{u}), F\left(t^{\prime}, x^{\prime}, \widetilde{u}\right)\right) \\
& \geq a|u-\widetilde{u}|-\frac{\alpha}{2} .
\end{aligned}
$$

In conclusion, combining (8) and (9) we get

$$
|u-\widetilde{u}| \leq \frac{\alpha}{a}+\frac{b \varepsilon^{\prime}}{2 a} \leq \frac{\varepsilon^{\prime}}{2}+\frac{b \varepsilon^{\prime}}{2 a}<\frac{(a+b)}{2 a} \varepsilon^{\prime}=\varepsilon .
$$

Remark 1. We have assumed, for simplicity, the continuity of $F$ with respect to the time $t$ and that the control set $U$ is independent on $(t, x)$. Indeed, for the validity of Theorem 1, we can assume only the measurability of $F$ with respect to $t$ and $U=U(t, x)$. The multivalued map $U:[0, T] \times \mathbf{R}^{n} \multimap W$, where $W$ is a compact subset of $\mathbf{R}^{m}$, is assumed to satisfy the following conditions: 
$\left(\mathrm{U}_{1}\right) U(t, x)$ is a nonempty convex closed set for any $(t, x) \in[0, T] \times \mathbf{R}^{n}$.

$\left(\mathrm{U}_{2}\right) U$ is measurable.

$\left(\mathrm{U}_{3}\right) U(t, \cdot)$ is lower semicontinuous for a.a. $t \in[0, T]$.

In fact, under these assumptions following the arguments employed in ([21], Theorem 3.4) and adapting the proof of Theorem 1 we can show that the regulation map $R$ has nonempty closed values, it is measurable with respect to $(t, x)$ and lower semicontinuous in $x$.

2. Some comments and remarks. Some comments on the assumptions are useful. First of all, observe that $\left(\mathrm{H}_{1}\right)$ and the Michael's selection theorem imply that $F$ has the continuous selection property. That is, for any $p_{0} \in F\left(t_{0}, x_{0}, u_{0}\right)$ with $\left(t_{0}, x_{0}, u_{0}\right) \in D$ there exists a continuous selection $f$ of $F$ such that $p_{0}=f\left(t_{0}, x_{0}, u_{0}\right)$. In fact, for any $\left(t_{0}, x_{0}, u_{0}\right) \in D$ and $p_{0} \in F\left(t_{0}, x_{0}, u_{0}\right)$, it is sufficient to consider the multivalued map $G: D \multimap \mathbf{R}^{n}$ defined as follows:

$$
G(t, x, u)= \begin{cases}F(t, x, u) & \text { for }(t, x, u) \neq\left(t_{0}, x_{0}, u_{0}\right) \\ \left\{p_{0}\right\} & \text { for }(t, x, u)=\left(t_{0}, x_{0}, u_{0}\right)\end{cases}
$$

which satisfies the assumptions of the Michael's selection theorem.

Furthermore, if we assume a more restrictive condition on the continuity of $F$ on $D$, then any continuous selection $f$ of $F$ can be expressed as a convex combination of extreme points of the set $F(t, x, u)$, (see [24]).

Concerning $\left(\mathrm{H}_{2}\right)$, observe that even if $F$ were a singlevalued continuous map condition $\left(\mathrm{H}_{2}\right)$ could not be weakened to the following (tangentiality) condition:

- for any $(t, x) \in[0, T] \times \mathbf{R}^{n}$ there exists $u \in U$ such that $F(t, x, u) \subset T(x)$.

In fact, in this case the map $R(t, x)$ could be not longer lower semicontinuous as the following simple example shows (see [13]).

ExAmple 1. Let $f:[0,2] \times[0,1] \times[0,1] \rightarrow \mathbf{R}$ be the map defined as follows

$$
f(t, x, u)= \begin{cases}\max \{1-u, t\}-1 & \text { for } t \leq 1 \\ \max \{u, 2-t\}-1 & \text { for } t \geq 1 .\end{cases}
$$

Then $f$ satisfies our conditions, $K=[0,1]$ and the feedback map $R:[0,2] \times[0,1] \multimap \mathbf{R}$ is given by

$$
R(t, x)= \begin{cases}\{0\} & \text { for } x=0 \text { and } t<1 \\ \{1\} & \text { for } x=0 \text { and } t>1 \\ {[0,1]} & \text { for } x=0 \text { and } t=1 \text { or } x>0\end{cases}
$$

which is not lower semicontinuous.

We exhibit now a class of uncertain control dynamics $F$ for which assumptions $\left(\mathrm{H}_{3}\right)$ is satisfied.

Example 2. Consider $F:[0, T] \times \mathbf{R}^{n} \times U \multimap \mathbf{R}^{n}$ of the form

$$
F(t, x, u)=\phi(t, x)+f(t, x) h(u)
$$

where 
(i) $\phi:[0, T] \times \mathbf{R}^{n} \multimap \mathbf{R}^{n}$ has nonempty closed convex values and it is bounded and continuous in the Hausdorff metric.

(ii) $f:[0, T] \times \mathbf{R}^{n} \rightarrow \mathbf{R}^{n}$ is a continuous function for which there exist constants $m_{1}, m_{2}>0$ such that

$$
m_{1} \leq|f(t, x)| \leq m_{2} \quad \text { for any }(t, x) \in[0, T] \times \mathbf{R}^{n} .
$$

(iii) $h: \mathbf{R}^{m} \rightarrow \mathbf{R}$ is a continuous map. Moreover, there exist constants $\lambda, \Lambda>0$ such that

$$
\lambda|u-v| \leq|h(u)-h(v)| \leq \Lambda|u-v|
$$

for any pair $u, v \in U$, with $U$ connected compact set of $\mathbf{R}^{m}$.

It is easy to verify that $F$ satisfies assumption $\left(\mathrm{H}_{3}\right)$.

Re mark 2. By Remark 1 and considering the restriction of $F$ to $[0, T] \times K$ it follows that Theorem 1 is a generalization of ([2], Theorem 3, p. 49) and ([1], Theorem 6.3.1) even in the singlevalued case. In fact, it reduces to this result if the dynamics is affine in $u$ and the process is autonomous. Moreover, observe that in the multivalued case even if $F$ is affine in the control we cannot use the argument employed in the proof of ([2], Theorem 3 p. 49) in order to prove our result. For instance, if $F(t, x, u)=\psi(t, x) u$, where $u \in \mathbf{R}$ and $\psi(t, x) \subset \mathbf{R}^{n}$ is a closed convex set, the set $\theta F(t, x, u)+(1-\theta) F(t, x, v)$ does not necessarily coincide with the set $F(t, x, \theta u+(1-\theta) v)$ unless $u \in U=[a, b], a>0$. Finally, in $\left(\mathrm{H}_{3}\right)$ we have assumed the existence of constants $a, b>0$ such that

$$
a|u-v| \leq d(F(t, x, u), F(t, x, v)) \leq b|u-v|
$$

for any $(t, x) \in[0, T] \times \mathbf{R}^{n}$ and any pair $u, v \in U$. This condition appears reasonable from the point of view of the effectiveness of the control variable $u$ on the dynamical system. In fact, the dynamics is sensitive with respect to the variations of the control via the parameter $a$. On the other hand, the parameter $b$ provides a necessary upper bound on this sensitivity.

Remark 3. Condition $\left(\mathrm{H}_{4}\right)$ is satisfied, for instance, if $K$ is a nonempty closed convex set or if $K$ is a proximate retract with $I=\mathbf{R}^{n}$.

3. Main result. In this section we state and prove the main result which solves the proposed problem. For this, we assume also the following condition.

$\left(\mathrm{H}_{5}\right)$ There exists a positive constant $M$ such that

$$
|F(t, x, u)| \leq M \quad \text { for any }(t, x, u) \in D .
$$

Here $|F(t, x, u)|=\sup \{|y| \mid y \in F(t, x, u)\}$.

We give in the sequel a definition and a result which is a direct consequence of ([10], Theorem 1).

Definition 6. Consider the cone

$$
\Gamma^{M}:=\left\{(t, x) \in \mathbf{R} \times \mathbf{R}^{n}|| x \mid \leq M t\right\} .
$$

We say that a map $f: \mathbf{R} \times \mathbf{R}^{n} \rightarrow \mathbf{R}^{n}$ is directionally $\Gamma^{M}$-continuous at a point $(\bar{t}, \bar{x})$ if and only if $f\left(t_{n}, x_{n}\right) \rightarrow f(\bar{t}, \bar{x})$ for every sequence $\left(t_{n}, x_{n}\right) \rightarrow(\bar{t}, \bar{x})$ with $\left(t_{n}-\bar{t}, x_{n}-\bar{x}\right) \in \Gamma^{M}$ 
for any $n \geq 1$. Moreover, we say that it is $\Gamma^{M}$-continuous on a set $Q \subset \mathbf{R}^{n+1}$ if it is $\Gamma^{M}$-continuous at every point $(\bar{t}, \bar{x}) \in Q$.

Proposition 2. Under assumptions $\left(H_{1}\right)-\left(H_{5}\right)$, the regulation map $R$ admits a $\Gamma^{M}$. continuous selection $\bar{u}$ defined on $[0, T] \times \mathbf{R}^{n}$.

Proposition 2 guarantees that any dynamics $f(t, x, \bar{u}(t, x))$ of $F(t, x, \bar{u}(t, x))$ is $\Gamma^{M_{-}}$ continuous with respect to $(t, x) \in[0, T] \times \mathbf{R}^{n}$. Observe that the Krasovskiil's regularization $K(g)(t, x)$ of $g(t, x):=f(t, x, \bar{u}(t, x))$ is not necessarily contained in $F(t, x, \bar{u}(t, x))$.

We prove now the main result.

THEOREM 2. Under assumptions $\left(H_{1}\right)-\left(H_{5}\right)$, any Krasovskiu's solution of the Cauchy problem

$$
\left\{\begin{array}{l}
\dot{x}=f(t, x, \bar{u}(t, x)) \\
x(0)=x_{0} \in K
\end{array}\right.
$$

satisfies $x(t) \in K$, for any $t \in[0, T]$.

Proof. First, we show that if $x(t), t \in[0, T]$, is an absolutely continuous function such that

$$
\left\{\begin{array}{l}
\dot{x}(t) \in K(g)(t, x(t)) \quad \text { for a.a. } t \in[0, T] \\
x(0)=x_{0}
\end{array}\right.
$$

where $x_{0} \in \mathbf{R}^{n}$, then $x(t), t \in[0, T]$, satisfies the Cauchy problem

$$
\left\{\begin{array}{l}
\dot{x}(t)=g(t, x(t)) \quad \text { for a.a. } t \in[0, T], \\
x(0)=x_{0} .
\end{array}\right.
$$

For this, we use the arguments of [11] and [14] which combine the $\Gamma^{M}$-continuity with standard techniques.

By means of Lusin's Theorem there exists $Q_{n} \subset[0, T], n \in \mathbf{N}$, measurable sets such that $\dot{x}$ restricted to $Q_{n}$ is continuous, $\dot{x}(t) \in K(g)(t, x(t))$ for any $t \in Q_{n}$ and $\mu\left([0, T] \backslash \cup_{n \in \mathrm{N}} Q_{n}\right)=0$. Moreover, the Lebesgue's density theorem (see [22]) ensures, for any $n \in \mathbf{N}$, the existence of a measurable set $N_{n} \subseteq Q_{n}$, with $\mu\left(N_{n}\right)=0$ such that any point $t \in Q_{n} \backslash N_{n}$ is a density point for $Q_{n}$. Let $t \in Q_{n} \backslash N_{n}$, we can find a sequence $\left\{t_{m}\right\} \subset Q_{n} \backslash N_{n}$ with $t_{m}>t$ and $t_{m} \rightarrow t$ decreasing as $m \rightarrow \infty$.

Hence $\dot{x}\left(t_{m}\right) \rightarrow \dot{x}(t)$. On the other hand $|x(t)-x(s)| \leq M|t-s|$ for all $t, s \in[0, T]$, since $|K(g)(t, x)| \leq M$ by $\left(\mathrm{H}_{5}\right)$, the fact that $g$ is a selection of $F$ and the definition of $K(g)$. Let $\varepsilon>0$. We have $\dot{x}\left(t_{m}\right) \in K(g)\left(t_{m}, x\left(t_{m}\right)\right) \subseteq g\left(t_{m}, x\left(t_{m}\right)\right)+\frac{\varepsilon}{2} B_{1}$ for any $m \in \mathbf{N}$. But $g$ is $\Gamma^{M}$-continuous in $[0, T] \times \mathbf{R}^{n}$, hence there exists $m_{0}(\varepsilon) \in \mathbf{N}$ such that for any $m \geq m_{0}$ we have $\left|g\left(t_{m}, x\left(t_{m}\right)\right)-g(t, x(t))\right|<\varepsilon / 2$, that is

$$
g\left(t_{m}, x\left(t_{m}\right)\right) \in g(t, x(t))+\frac{\varepsilon}{2} B_{1} .
$$

Therefore for $m \geq m_{0}$ we have $\dot{x}\left(t_{m}\right) \in g(t, x(t))+\varepsilon B_{1}$ and so $\dot{x}(t) \in g(t, x(t))+\varepsilon B_{1}$.

For $\varepsilon \rightarrow 0$ we get $\dot{x}(t)=g(t, x(t)), x(0)=x_{0}$ for all $t \in \widehat{Q}=\cup_{n \in \mathrm{N}}\left(Q_{n} \backslash N_{n}\right)$ with $\mu([0, T] \backslash \widehat{Q})=0$.

In conclusion, $x(t)$ is an absolutely continuous function such that

$$
\dot{x}(t) \in T(x(t)) \quad \text { for a.a. } t \in[0, T] .
$$


Now if we take $x_{0} \in K$ then $x(t) \in K$ for a.a. $t \in[0, T]$. To show this, let $t \in[0, T]$ such that there exists $\dot{x}(t)$ and $\dot{d}(t)$, where $d(t):=\operatorname{dist}(x(t), K)$ is absolutely continuous. Consider $x(t+\tau)$, with $\tau>0$, there exists $\varepsilon(\tau)$ with $\varepsilon(\tau) \rightarrow 0$ when $\tau \rightarrow 0^{+}$such that

$$
x(t+\tau)=x(\tau)+\tau \dot{x}(t)+\tau \varepsilon(\tau) .
$$

Therefore

$$
\dot{d}(t)=\lim _{\tau \rightarrow 0^{+}} \frac{\operatorname{dist}(x(\tau)+\tau \dot{x}(t)+\tau \varepsilon(\tau), K)-\operatorname{dist}(x(t), K)}{\tau} .
$$

On the other hand by Proposition 1 we have $\dot{x}(t) \in T(x(t)) \subset \widehat{T}_{K}(x(t))$, thus $\dot{d}(t) \leq 0$ for a.a. $t \in[0, T]$. From this if $t \in[0, T]$ is such that $d(t)>0$ we have

$$
0<d(t)=\int_{0}^{t} \dot{d}(s) d s \leq 0
$$

which is a contradiction. This concludes the proof.

Remark 4. From the proof of Theorem 2 it turns out that any Krasovskiı's solution $x(t)$ of (2), whenever $f(t, x, \bar{u}(t, x)) \in F(t, x, \bar{u}(t, x))$, is such that the function $\operatorname{dist}(x(t), K)$ is nonincreasing in $[0, T]$. Therefore, if the initial condition $x(0)=x_{0}$ is sufficiently close to $K$, say $\operatorname{dist}\left(x_{0}, K\right)<\varepsilon$, then $\operatorname{dist}(x(t), K)<\varepsilon$ for any $t \in[0, T]$. Furthermore, if $K$ is bounded and the initial conditions $x_{0}$ are taken in a bounded neighbourhood $V \subset B(0, r)$ of $K$, then condition $\left(\mathrm{H}_{5}\right)$ can be replaced by the weaker condition:

$\left(\mathrm{H}_{5}\right)^{\prime}$ there exists a constant $M>0$ such that

$$
|F(t, x, u)| \leq L
$$

for any $t \in[0, T]$, any $|x|<2 r$ and any $u \in U$.

Observe that if $F$ satisfies $\left(\mathrm{H}_{1}\right)$ and $F(t, x, u)$ is a bounded set for any $(t, x, u) \in D$ then $\left(\mathrm{H}_{5}\right)^{\prime}$ is satisfied.

In Remark 1 we pointed out that if $F$ is Carathéodory and $U=U(t, x)$ satisfies $\left(\mathrm{U}_{1}\right)$, $\left(\mathrm{U}_{2}\right)$ and $\left(\mathrm{U}_{3}\right)$ then the regulation map $R$ is measurable in $t$ and lower semicontinuous in $x$ with nonempty closed values. Thus, if we introduce for $\bar{u}(t, x)$ the notion of ScorzaDragoni $\Gamma^{M}$-continuity (see e.g. [14]) we can prove Theorem 2 under these assumptions. Furthermore, under some regularity assumption, we could also allow the set $K$ to depend on $t$, and the existence of periodic solutions of (2) in $K$ could be also investigated.

4. An application. An important control problem for system (1) that we can tackle by means of the approach presented in this paper is illustrated in the sequel.

Consider two control systems

$$
\dot{x} \in F(t, x, u), \quad t \in[0, T], u \in U \subset \mathbf{R}^{m}, x \in \mathbf{R}^{n}
$$

and

$$
\dot{y}=\psi(t, y, v), \quad t \in[0, T], v \in V \subset \mathbf{R}^{m}, y \in \mathbf{R}^{n},
$$

where $U, V$ are connected compact sets of $\mathbf{R}^{n}$ and $\psi$ is continuous.

The initial state $x(0)=x_{0}$ is also uncertain, but bounded, i.e., $x_{0} \in B(0, r), r>0$. The initial condition $y(0)=y_{0}$ is taken in a given ball $B(0, \rho), \rho>0$. 
Introduce now a multivalued map $Q: \mathbf{R}^{n} \multimap \mathbf{R}^{n}$, where the domain represents the states $y$ and the range represents the states $x$. Assume the following conditions

$\left(\mathrm{Q}_{1}\right) Q(y)$ is a non empty convex compact set of $\mathbf{R}^{n}$ for any $y \in \mathbf{R}^{n}$ and $Q$ is upper semicontinuous.

$\left(\mathrm{Q}_{2}\right) \operatorname{Graph}(Q)$ is a convex set of $\mathbf{R}^{n} \times \mathbf{R}^{n}$ containing the set $B(0, \rho) \times B(0, r)$.

The map $Q^{-1}$ is often called the observation map.

We consider the following problem.

(P) Given a state-control pair $(y(t), v(t))$ of $(\psi)$, the problem that we want to solve is that of determining a feedback control $\bar{u}(t, x),(t, x) \in[0, T] \times \mathbf{R}^{n}$ such that for any dynamics $f(t, x, \bar{u}(t, x)) \in F(t, x, \bar{u}(t, x))$ and for any $x_{0} \in B(0, r)$ we have $x(t) \in Q(y(t))$ for any $t \in[0, T]$, where $x(t)$ is any Krasovskiu's solution of the Cauchy problem

$$
\left\{\begin{array}{l}
\dot{x}=f(t, x, \bar{u}(t, x)), \\
x(0)=x_{0} .
\end{array}\right.
$$

In other words, given a nonlinear control model $(\psi)$ we want to find a feedback control $\bar{u}(t, x)$ under which any solution, in the Krasovskil sense, of $(\mathrm{F})$ is such that the pair $(y(\cdot), x(\cdot))$ is viable in the graph of $Q$.

Let $K:=\operatorname{Graph} Q$. Since $K$ is a closed convex subset of $\mathbf{R}^{n} \times \mathbf{R}^{n}$ there exists a continuous (non-expansive) projector $\pi_{K}: \mathbf{R}^{n} \times \mathbf{R}^{n} \rightarrow K$. Define now the regulation $\operatorname{map} R_{Q}:[0, T] \times \mathbf{R}^{n} \times \mathbf{R}^{n} \times V \multimap U$ as follows.

$$
R_{Q}(t, x, y, v)=\left\{u \in U \mid(\psi(t, y, v), F(t, x, u)) \subset T_{K}\left(\pi_{K}(y, x)\right)\right\}
$$

if $v \in V$ and $R_{Q}(t, x, y, v)=\emptyset$ if $v \notin V$.

We have the following result.

Proposition 3. Under assumptions $\left(\mathrm{H}_{1}\right),\left(\mathrm{H}_{2}\right),\left(\mathrm{H}_{3}\right),\left(\mathrm{H}_{5}\right)$ and $\left(\mathrm{Q}_{1}\right),\left(\mathrm{Q}_{2}\right)$ problem (P) is solvable.

Proof. For any fixed state-control pair $(y(t), v(t))$ of the reference model $(\psi)$, by Theorem 1 we get that the map

$$
(t, x) \rightarrow \widetilde{R}_{Q}(t, x):=R_{Q}(t, x, y(t), v(t))
$$

is lower semicontinuous with nonempty closed values. Then we can apply the first part of the proof of Theorem 2 to conclude that

$$
(\dot{y}(t), \dot{x}(t)) \in T_{K}\left(\pi_{K}(y(t), x(t))\right) .
$$

On the other hand $\left(y_{0}, x_{0}\right) \in K$ and so using the second part of the proof of Theorem 2 we obtain that

$$
(y(t), x(t)) \in K \quad \text { for any } t \in[0, T] .
$$

Observe that the condition

$$
(\psi(t, y, v), F(t, x, u)) \subset T_{K}\left(\pi_{K}(y, x)\right)
$$


can be rewritten in terms of the contingent derivative $D Q(y, x)$ of the map $Q$ as follows:

$$
F(t, x, u) \subset D Q(y, x)(\psi(t, y, v)) .
$$

In fact

$$
\text { Graph } D Q(y, x):=T_{\operatorname{Graph} Q}(y, x) .
$$

A similar control problem was treated in [19] and [20] (see also [7], [8]) by means of an approach based on the theory of variable structure systems. More precisely, by introducing a sliding manifold as the zeros of a non differentiable function $s$ and using the Clarke's generalized gradient of $s$, conditions ensuring the asymptotic linearization of an uncertain control system of the form (F) were given.

Finally, tracking problems concerning singlevalued control systems affine in the controls are considered in ([1], Chapt. 8, Sect.2).

\section{References}

[1] J. P. Aubin, Viability Theory, Birkhäuser-Verlag, Berlin, 1991.

[2] J. P. Aubin and A. Cellina, Differential Inclusions, Springer-Verlag, 1984.

[3] J. P. A ubin and H. Frankowska, Set-Valued Analysis, Birkhäuser-Verlag, Berlin, 1990.

[4] J. P. Aubin and H. Frankowska, Observability of systems under uncertainty, SIAM J. Control and Optimization 27 (5) 1989, 949-975.

[5] B. R. Barmish, Stabilization of uncertain systems via linear control, IEEE Trans. Autom. Contr. AC-28 (8) 1983, 848-850.

[6] B. R. Barmish, M. Corless and G. Leitmann, A new class of stabilizing controllers for uncertain dynamical systems, SIAM J. Control and Optim. 21 (2) 1983, 246-255.

[7] G. Bartolini and T. Zolezzi, Asymptotic linearization of uncertain systems by variable structure control, System, and Control Letters 10 1988, 111-117.

[8] G. Bartolini and T. Zolezzi, Some new application of V.S.S. theory to the control of uncertain nonlinear systems, Proc. 27-th IEEE Conference on Decision and Control, Austin 1988.

[9] R. Bielawski, L. Górniewicz and S. Plaskacz, Topological approach to differential inclusions on closed subsets of $R^{n}$, Dynamics Reported N.S. 1 1992, 225-250.

[10] A. Bressan, Directionally continuous selections and differential inclusions, Funkc. Ekv. 31 1988, 459-470.

[11] A. Bressan, Upper and lower semicontinuous differential inclusions: a unified approach, Nonlinear Controllability and Optimal Control, H. Sussmann Ed., M. Dekker, 1988, 2131.

[12] M. J. Corless and G. Leitmann, Continuous state feedback guaranteeing uniform ultimate boundedness for uncertain dynamic systems, IEEE Trans. Autom. Contr. AC-26 (25) 1981, 1139-1144.

[13] L. Górniewicz, P. Nistri and P. Zecca, Control problems in closed subset of $R^{n}$ via feedback controls, TMNA 2 1993, 163-178.

[14] S. Hu and N. S. Papageorgiou, On the existence of periodic solutions of nonconvex valued differential inclusions in $R^{n}$, Technical Report.

[15] I. Joong Ha and E. G. Gilbert, Robust tracking in nonlinear systems, IEEE Trans. Autom. Contr. AC-32 (9) 1987, 763-771. 
[16] M. Kisielewicz, Differential Inclusions and Optimal Control, PWN-Polish Scientific Publishers, Warszawa \& Kluwer Academic Publishers, Dordrecht, Boston, London, 1991.

[17] A. B. Kurzhanskiŭ, Control and observation under conditions of uncertainty, Nauka, Russia, 1997.

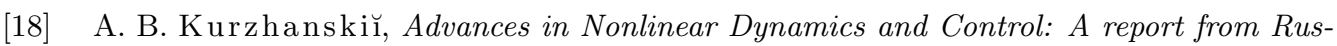
sia, Verlag, Berlin, 1993.

[19] J. W. Macki, P. Nistri and P. Zecca, A tracking problem for uncertain vector systems, Nonlinear Analysis TMA 14 1990, 319-328.

[20] J. W. Macki, P. Nistri and P. Zecca, Corrigendum: A tracking problem for uncertain vector systems, Nonlinear Analysis TMA 20 1993, 191-192.

[21] P. Nistri, V. Obukhovskiǔ and P. Zecca, Viability for feedback control systems in Banach spaces via Carathéodory closed-loop controls, Diff. Eqns and Dyn. Sys., (to appear).

[22] J. Oxtoby, Measure and Category, Springer-Verlag, New York, 1971.

[23] S. Plaskacz, Periodic solution of differential inclusions on compact subset of $R^{n}$, J. Math. Anal. and Appl. 148 1990, 202-212.

[24] G. Stefani and P. Zecca, Properties of convex sets with application to differential theory of multivalued functions, Nonlinear Analysis TMA 2 1978, 581-593. 